\title{
Study galactic cosmic ray modulation with AMS-02 observation
}

\author{
Xiaojian Song, ${ }^{a, *} \mathbf{X i ~ L u o}{ }^{a}$ and Weiwei $\mathbf{X u}^{a, b}$ \\ ${ }^{a}$ Shandong Institute of Advanced Technology (SDIAT), Jinan, Shandong, China. \\ ${ }^{b}$ Shandong University (SDU), Jinan, Shandong, China. \\ E-mail: xiaojian.song@iat.cn, xi.luo@iat.cn
}

The accurate measurements of the galactic cosmic ray (GCR) fluxes as function of time and energy by the Alpha Magnetic Spectrometer (AMS) give us unique information to study the dynamics of solar modulation. To reproduce the observed data, a 3D time-dependent numerical model is established. The input parameters in the model (solar wind speed, tilt angle, magnetic intensity and polarity) are obtained by the observation near the Earth at the right time. The time varying parameters (diffusion coefficient, drift coefficient) is constrained by the proton and helium spectrum pair and obtained by Markov Chain Monte Carlo (MCMC) method. With the obtained coefficients, the proton and helium spectrum are reproduced with normalized $\chi^{2}$ mainly less than 1. Finally, the time variations of $\mathrm{He} / \mathrm{P}$ at rigidity of $2.035 \mathrm{GV}$ (fit well with AMS-02 observation) and $0.5 G V$ are studied. They show different behavior: The former one is mainly caused by the dependence of modulation on the particle mass-to-charge ratio, the latter one is mainly caused by the difference of particle's local interstellar spectrum.

$37^{\text {th }}$ International Cosmic Ray Conference (ICRC 2021)

July 12th - 23rd, 2021

Online-Berlin, Germany

\footnotetext{
${ }^{*}$ Presenter
} 


\section{Introduction}

Galactic Cosmic Rays (GCRs), which are charged, energetic particles originated outside the solar system, are accelerated by the energetic processes in the interstellar medium [e.g., 1], show an isotropic distribution outside the heliopause. When entering the heliosphere, the flux of GCRs and it's spectrum shape are modulated by the disturbed solar wind with an embedded magnetic fields, which cause the so called solar modulation effect [2]. With the change of solar activity, the GCR flux show an 11-year cycle and anti-correlated with the sunspot number [e.g., 3, 4].

The accurate measurements of proton and helium spectrum by AMS-02 gives an unprecedented opportunity to study the difference of modulation between proton and helium, which is called the dependency of modulation on mass-to-charge ratio $(A / Z)$. [5] studied the P/He observed by AMS02 from 2011 to 2017 and found that the decreases of P/He after solar polarity reversal is mainly caused by the dependence of modulation on $A / Z$. [6] reproduced the proton and helium data observed by PAMELA from 2006 to 2009, and made a comprehensive study of the influence of different local interstellar spectrum (LIS) and $A / Z$ on the time variation of $\mathrm{P} / \mathrm{He}$ in this time interval.

In this work, a time-dependent numerical model is established, and the proton and helium spectrum pair observed by AMS-02 from 2011 to 2017 are used to constrain the parameters in this model. In Section 2, the time-dependent model is described in detail along with the heliosphere, drift and diffusion models. The model result and discussion is placed in Section 3. Summary and conclusions are presented in Section 4.

\section{The numerical model}

The numerical model is based on the well known Parker transport equation [TPE; 2]:

$$
\frac{\partial f}{\partial t}=-\left(\vec{V}_{s w}+\vec{V}_{d}\right) \cdot \nabla f+\nabla \cdot\left(\stackrel{\leftrightarrow}{K}_{s} \cdot \nabla f\right)+\frac{1}{3}\left(\nabla \cdot \vec{V}_{s w}\right) \frac{\partial f}{\partial \ln p},
$$

where $f(\vec{r}, p, t)$ is the GCR phase space distribution function, $\vec{r}$ is the position, $p$ is the momentum, and $t$ is the time. $f(\vec{r}, p, t)$ is related to the differential intensity $j(E)$ by $j=p^{2} f . \vec{V}_{s w}$ is the solar wind speed. $\vec{V}_{d}$ is the pitch angle averaged drift velocity, $\stackrel{\leftrightarrow}{K}_{s}$ is the diffusion tensor. In this work, TPE is solved by means of time-backward stochastic differential equations (SDE) [e.g., 7-9].

Drift caused by large scale heliospheric magnetic field (HMF) can be expressed by:

$$
\vec{V}_{d}=\nabla \times\left(K_{A} \frac{q R \beta}{3 B} \frac{\left(R / R_{0}\right)^{2}}{1+\left(R / R_{0}\right)^{2}} \frac{\vec{B}}{B}\right),
$$

where $K_{A}$ is a constant, range from 0 to $1, K_{A}=1$ describes full gradient, curvature and current sheet drifts, $q$ is the particle charge sign, $\beta=v / c$ is the ratio between the speed of the particle $v$ and that of light, $R$ is the rigidity of particle, $R_{0}=0.9 G V . B$ is the magnitude HMF.

Irregularity of HMF in small scale caused the diffusion of GCR parallel and perpendicular to the background large scale HMF. Many theories have been developed to describe the properties of diffusion [e.g., 10-12]. But there are still obstacles to overcome for directly adapting to numerical 
model. Therefore, a widely used empirical formula is used in this work [e.g., 13-15]:

$$
\begin{aligned}
K_{\|} & =K_{0} \beta\left(\frac{B_{e q}}{B}\right)\left(\frac{R}{1 G V}\right)^{b}\left(\frac{\left(\frac{R}{1 G V}\right)^{d}+\left(\frac{R_{k}}{1 G V}\right)^{d}}{1+\left(\frac{R_{k}}{1 G V}\right)^{d}}\right)^{\frac{c-b}{d}}, \\
K_{\perp, r} & =0.02 K_{\|}, \\
K_{\perp, \theta} & =\left\{A^{+} \mp A^{-} \tanh \left[8\left(\theta_{A}-90^{\circ}\right) \pm \theta_{F}\right]\right\} K_{\perp, r},
\end{aligned}
$$

where, $K_{0}$ is a constant in units of $10^{22} \mathrm{~cm}^{2} \mathrm{~s}^{-1}$, with the rest of the equation written to be dimensionless with $B_{e q}$ is the HMF measured near the Earth. This formula is in double power law form. The slope of rigidity dependence is $b$ and $c$ when the particle rigidity below and above than $R_{k}$, respectively. $d$ determines the smoothness of the transition, and it is set equal to 3 for simplicity. $A^{ \pm}=(d \pm 1) / 2, \theta_{F}=35^{\circ}, \theta_{A}=\theta$ for $\theta \leq 90^{\circ}$ but $\theta_{A}=180^{\circ}-\theta$ with $\theta>90^{\circ}$.

Input parameters of time-dependent numerical model, solar polarity $(A)$, current sheet tilt angle $(\alpha)$, solar wind velocity $\left(V_{0}\right)$ and magnetic filed strength $\left(B_{0}\right)$, are related to the actual measurement near the Earth at the right time. For a pseudo-particle located at $r$ at time $t$, the interplanetary conditions it experienced come from the Sun at time $t_{0}=t-\left(r-r_{0}\right) / V_{s w}$. The time varying coefficients $K_{A}, K_{0}, b, c, R_{k}$, are determined in each case using Markov Chain Monte Carlo method, which is widely used in other research area [e.g., 16-18]. The Metropolis-Hastings algorithm is used to implement the MCMC sampler to generate large sequences of random samples. After a large sample, chain accordance with the posterior probability distribution is eventually obtained.

The local interstellar spectrum (LIS) of proton and helium, which are the boundary (heliopause) condition of numerical model, are given in detail by [5]. As the $\mathrm{He} / \mathrm{He} 4$ in [5] does not accord with the actual observation made by AMS-02 [19], the sum of He3 and He4 flux in [5] is taken as the total flux of helium and it is redistributed as fluxes of $\mathrm{He} 3$ and $\mathrm{He} 4$ according to the following ratio measured by AMS-02:

$$
\frac{j_{h p, \text { He3 }}}{j_{h p, H e 4}}= \begin{cases}0.1476(R / 4 G V)^{-0.294} & R>4 G V \\ 0.1476(R / 4 G V)^{-0.21} & R \leq 4 G V\end{cases}
$$

\section{Result and discussion}

\subsection{Drift and diffusion coefficients over time}

The numerical model is applied to all 79 cases (proton and helium spectrum pair) observed by AMS-02. For each spectrum pair, we calculate the sum of square deviation to the measured spectrum pair and choose the coefficient vector with the smallest $\chi^{2}$ as the best-fit coefficients. The top five panels in Figure 1 shows the best-fit diffusion and drift coefficients in each case, the last panel show the normalized $\chi^{2}$ between observation and model result using the best-fit parameters. In most cases, the $\chi^{2} / d o f$ is less than 1 , indicating a good agreement between model result and observations.

The breakpoint $\left(R_{k}\right)$ in Equation 3 vary considerably from case to case, making it difficult to discern any clear time-dependent pattern. Slope of rigidity dependence $b$ is smaller than $c$ before 2016 making Equation 3 concave upward. After 2016, Equation 3 become concave downward. 


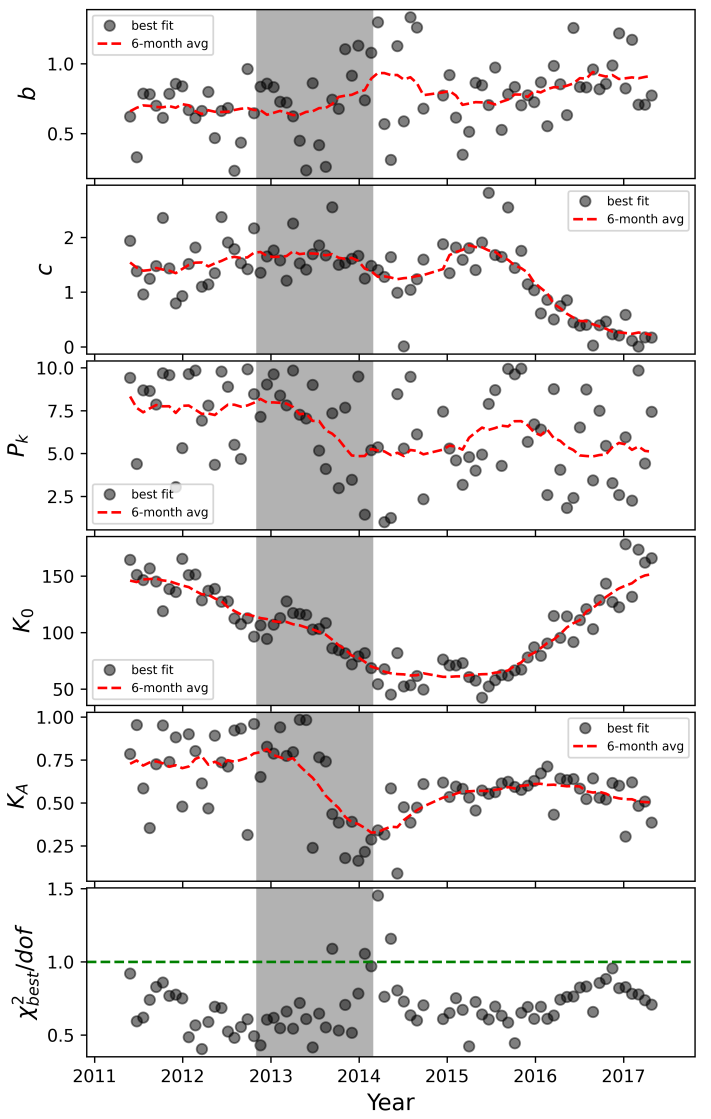

Figure 1: The top five panels show the drift and diffusion coefficients from 2011 to 2017, the last panel shows the normalized $\chi^{2}$ between model result and observation.

The normalization of diffusion $K_{0}$ (indicator of particle parallel mean free path $\lambda_{\|}$) shows a clear time-dependent pattern: it decreases to the minimum value after solar polarity reversal (SPR, delimited by gray area), increases again until 2017. The time variation of $K_{0}$ is similar to that calculated by [15], but for electron and positron. As we all know, diffusion of particle is caused by the turbulence of heliosphere magnetic field in small scale. [20] shows that the magnetic turbulence magnitude increases with the increase of solar activity (indicated by sunspot number). So, it is not surprising that the diffusion coefficient for leptons and nuclei have similar variation pattern. The drift coefficient $K_{A}$ is more scattered before SPR than that after SPR. The mean value of $K_{A}$ before 2013 is significantly larger than that after 2013, it decreases remarkably during SPR time interval and increases slightly after SPR.

\section{2 $\mathrm{He} / \mathrm{P}$ over Time}

In SDE method, the phase space distribution at Earth with momentum $p$ is an average of its values outside the heliopause, which can be described by:

$$
\frac{j(R)}{R^{2} \beta}=\frac{1}{N} \sum_{i=1}^{N} \frac{j_{h p}\left(R_{i}\right)}{R_{i}^{2} \beta_{i}}=\frac{j_{h p}\left(R_{h p}\right)}{R_{h p}^{2} \beta_{h p}} .
$$




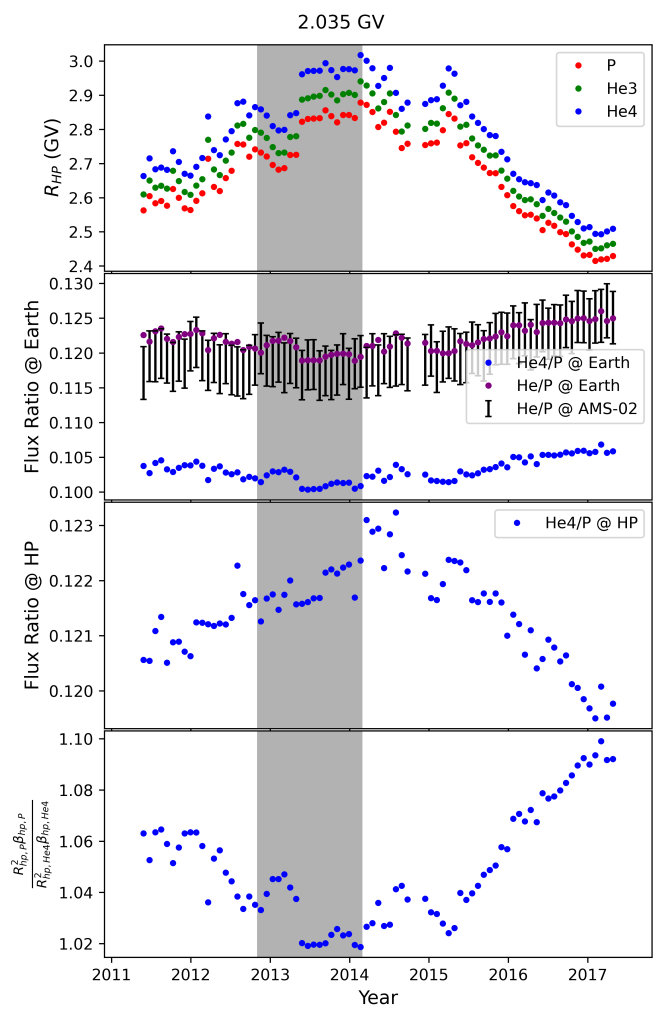

(a)

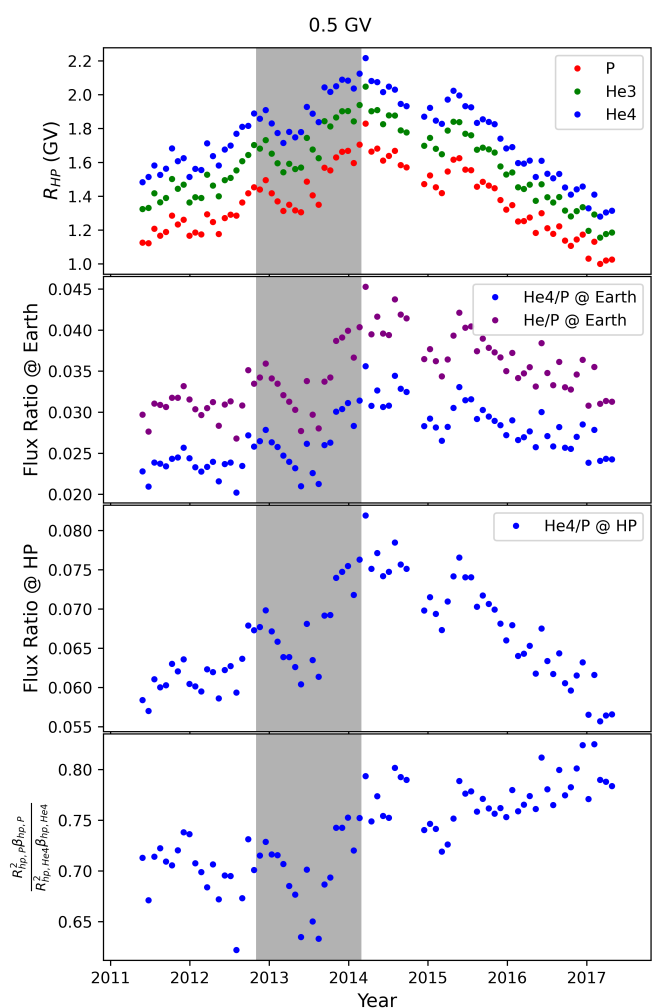

(b)

Figure 2: The effect of each part in Equation 6 on the flux ratio of helium and proton observed near the Earth at $R=2.035 G V$ (left) and $R=0.5 G V$ (right).

Then we can define an effective rigidity $R_{h p}$ which meets the requirement in Equation 5, which correspond to the average rigidity of pseudo-particle, which starts at rigidity equal to $R$ near the earth, when they exit the simulation domain. So the ratio of helium to proton flux with the same rigidity, $R$, can be written as:

$$
\frac{j_{H e *}(R)}{j_{P}(R)}=\frac{j_{h p, H e *}\left(R_{h p, H e *}\right)}{j_{h p, P}\left(R_{h p, P}\right)} \frac{R_{h p, P}^{2}}{R_{h p, H e *}^{2}} \frac{\beta_{h p, P}}{\beta_{h p, H e *}} \frac{\beta_{H e *}}{\beta_{P}},
$$

where $H e *$ denotes the isotopes of helium (He3 or He4). The right side of Equation 6 can be divided into 3 parts: $j_{h p, H e *} / j_{h p, P}$ denotes the influence of LIS's difference between helium and proton, $\left(R_{h p, P}^{2} \beta_{h p, P}\right) /\left(R_{h p, H e *}^{2} \beta_{h p, H e *}\right)$ denotes the dependence of solar modulation process on $A / Z$, the last part is a constant and does not change with time.

Figure 2(a) shows the time variation of each part in Equation 6 for $R=2.035 G V$. It can be seen in the second panel that the He/P calculated by our model fit well with that measured by AMS-02. For the particle ( $\mathrm{P}, \mathrm{He} 3$ and $\mathrm{He} 4$ ) measured at the Earth with $R=2.035 G V$, the corresponding effective rigidity outside the heliopause (see in the first panel) show the same time variation: increases to the maximum value $(\sim 3.0 \mathrm{GV})$ at the end of SPR, and decreases again. The value of He3 is always larger than that of $\mathrm{P}$, but smaller than that of $\mathrm{He} 4$. The third panel 
shows that the time variation pattern of LIS's ratio is similar to that of $R_{h p}$. Though the LIS's ratio is implicitly dependent on $A / Z$ through the difference of $R_{h p, P}$ and $R_{h p, H e *}$, LIS'a ratio has the similar variation trend under the same effective rigidity $R_{h p, P}$ or $R_{h p, H e *}$ (not show here). So, this implicit dependency on $A / Z$ has little effect on the overall variation of LIS's ratio. The last panel show that the second part of Equation 6 has opposite variation trend with $R_{h p}$ and have the similar variation trend with $\mathrm{He} / \mathrm{P}$ near the Earth. The amplitude of variation in this part (9.8\%) is larger than that of the first part (3.4\%). Therefore, the time variation of He/P observed near the Earth with $R=2.035 G V$ is not originated from the P, He LIS's difference but from the solar modulation effect related to the mass-to-charge ratio.

According to [5], the He/P outside the heliopause increases with the rigidity, and the slope is much larger when $R<2 G V$ than that when $R>2 G V$. Our simulation result shows that the dependence of solar modulation on $A / Z$ is the main factor caused the time variation of He/P near the Earth for the particle with rigidity equal to $2.035 \mathrm{GV}$. So, it is necessary to do a comparative research for particle with rigidity less than $2.035 \mathrm{GV}$.

Figure 2(b) is similar to Figure 2(b), but for particle with $R=0.5 G V$. In this case, $R_{h p}$ is mainly less than $2 G V$ and the variation amplitude is large enough, so the variation of first part in Equation 6 is larger than that of second part. The LIS's difference is the main factor leading to the variation of $\mathrm{He} / \mathrm{P}$ near the Earth at $R=0.5 G V$.

\section{Summary and Conclusion}

In this work, the proton and helium data measured by AMS-02 below $10 G V$ are utilized combined to get the drift and diffusion parameters of solar modulation from 2011 to 2017. The diffusion coefficient shows a clear anti-correlation with the variation of solar activity.

The proton and helium spectrum are reproduced in high precision by the same set of parameters. It is worthwhile to study the time variation of the flux ratio of helium to proton. As for the GCR solar modulation process, from the theoretical perspective, proton differs from helium in $A / Z$ and LIS. Our result show that the variation of $\mathrm{He} / \mathrm{P}$ at some high rigidity level is mainly caused by the dependence of modulation on $A / Z$. But at low rigidity, e.g., $0.5 G V$, the influence of LIS's difference is dominating.

\section{References}

[1] L.A. Fisk and G. Gloeckler, Acceleration of galactic cosmic rays in the interstellar medium, The Astrophysical Journal 744 (2012) 127.

[2] E.N. Parker, The passage of energetic charged particles through interplanetary space, Planetary and Space Science 13 (1965) 9.

[3] M. Potgieter, Solar modulation of cosmic rays, Living Reviews in Solar Physics 10 (2013).

[4] E. Ross and W.J. Chaplin, The behaviour of galactic cosmic-ray intensity during solar activity cycle 24, Sol Phys 294 (2019) 8. 
[5] C. Corti, M.S. Potgieter, V. Bindi, C. Consolandi, C. Light, M. Palermo et al., Numerical modeling of galactic cosmic-ray proton and helium observed by ams-02 during the solar maximum of solar cycle 24, The Astrophysical Journal 871 (2019) 253.

[6] M.D. Ngobeni, O.P.M. Aslam, D. Bisschoff, M.S. Potgieter, D.C. Ndiitwani, M. Boezio et al., The $3 d$ numerical modeling of the solar modulation of galactic protons and helium nuclei related to observations by pamela between 2006 and 2009, Astrophysics and Space Science 365 (2020) 182.

[7] M. Zhang, A markov stochastic process theory of cosmic-ray modulation, The Astrophysical Journal 513 (1999) 409.

[8] Z.N. Shen, G. Qin, P. Zuo and F. Wei, Modulation of galactic cosmic rays from helium to nickel in the inner heliosphere, The Astrophysical Journal 887 (2019) 132.

[9] X. Luo, M. Zhang, X. Feng, M.S. Potgieter, F. Shen and G. Bazilevskaya, A numerical study of the effects of corotating interaction regions on cosmic-ray transport, The Astrophysical Journal 899 (2020) 90.

[10] J.R. Jokipii, Cosmic-ray propagation. i. charged particles in a random magnetic field, The Astrophysical Journal 146 (1966) 480.

[11] W.H. Matthaeus, G. Qin, J.W. Bieber and G.P. Zank, Nonlinear collisionless perpendicular diffusion of charged particles, The Astrophysical Journal 590 (2003) L53.

[12] G. Qin, Nonlinear parallel diffusion of charged particles: Extension to the nonlinear guiding center theory, The Astrophysical Journal 656 (2007) 217.

[13] M.S. Potgieter, E.E. Vos, R. Munini, M. Boezio and V.D. Felice, Modulation of galactic electrons in the heliosphere during the unusual solar minimum of 2006-2009: A modeling approach, The Astrophysical Journal $\mathbf{8 1 0}$ (2015) .

[14] D. Bisschoff, M.S. Potgieter and O.P.M. Aslam, New very local interstellar spectra for electrons, positrons, protons, and light cosmic ray nuclei, The Astrophysical Journal 878 (2019) .

[15] O.P.M. Aslam, D. Bisschoff, M.D. Ngobeni, M.S. Potgieter, R. Munini, M. Boezio et al., Time and charge-sign dependence of the heliospheric modulation of cosmic rays, The Astrophysical Journal 909 (2021) .

[16] A. Putze, L. Derome, D. Maurin, L. Perotto and R. Taillet, A markov chain monte carlo technique to sample transport and source parameters of galactic cosmic rays, Astronomy I\& Astrophysics 497 (2009) 991.

[17] J. Liu, Q. Yuan, X. Bi, H. Li and X. Zhang, Markov chain monte carlo study on dark matter property related to the cosmice \pm excesses, Physical Review D 81 (2010) . 
[18] J. Feng, N. Tomassetti and A. Oliva, Bayesian analysis of spatial-dependent cosmic-ray propagation: Astrophysical background of antiprotons and positrons, Physical Review D 94 (2016) .

[19] M. Aguilar, L. Ali Cavasonza, G. Ambrosi, L. Arruda, N. Attig, A. Bachlechner et al., Properties of cosmic helium isotopes measured by the alpha magnetic spectrometer, Physical Review Letters 123 (2019).

[20] Z.N. Shen and G. Qin, Modulation of galactic cosmic rays in the inner heliosphere over solar cycles, The Astrophysical Journal 854 (2018) .

[21] D.C. Ndiitwani, S.E.S. Ferreira, M.S. Potgieter and B. Heber, Modelling cosmic ray intensities along the ulysses trajectory, Annales Geophysicae 23 (2005) 1061. 